\title{
Gap Symmetry of Superconductivity in $\mathrm{UPd}_{2} \mathrm{Al}_{3}$
}

\author{
H. Won, ${ }^{1}$ D. Parker,${ }^{2}$ K. Maki,${ }^{2}$ T. Watanabe,${ }^{3}$ K. Izawa,${ }^{3}$ and Y. Matsuda ${ }^{3}$ \\ ${ }^{1}$ Department of Physics, Hallym University, Chuncheon 200-702, South Korea \\ ${ }^{2}$ Department of Physics and Astronomy, \\ University of Southern California, Los Angeles, CA 90089-0484 USA \\ ${ }^{3}$ Institute for Solid State Physics, University of Tokyo, \\ Kashiwanoha 5-1-5, Kashiwa,Chiba 277-8581, Japan
}

(Dated: July 13, 2018)

\begin{abstract}
The angle dependent thermal conductivity of the heavy-fermion superconductor $\mathrm{UPd}_{2} \mathrm{Al}_{3}$ in the vortex state was recently measured by Watanabe et al. Here we analyze this data from two perspectives: universal heat conduction and the angle-dependence. We conclude that the superconducting gap function $\Delta(\mathbf{k})$ in $\mathrm{UPd}_{2} \mathrm{Al}_{3}$ has horizontal nodes and is given by $\Delta(\mathbf{k})=$ $\Delta \cos (2 \chi)$, with $\chi=c k_{z}$.

PACS numbers:
\end{abstract}




\section{Introduction}

Since the discovery of the heavy-fermion superconductor $\mathrm{CeCu}_{2} \mathrm{Si}_{2}$ in 1979[1] the gap symmetries of unconventional superconductors have become a central issue in condensedmatter physics [2]. In the last few years, the angle-dependent magnetothermal conductivity in the vortex state of nodal superconductors has been established as a powerful technique to address the gap symmetry. This is in part due to the theoretical understanding of the quasiparticle spectrum in the vortex state of nodal superconductors, following the path-breaking work by Volovik [3, 4, 5]. Using this approach, Izawa et al have succeeded in identifying the gap symmetries of superconductivity in $\mathrm{Sr}_{2} \mathrm{RuO}_{4}, \mathrm{CeCoIn}_{5}, \kappa-(\mathrm{ET})_{2} \mathrm{Cu}(\mathrm{NCS})_{2}, \mathrm{YNi}_{2} \mathrm{~B}_{2} \mathrm{C}$, and $\mathrm{PrOs}_{4} \mathrm{Sb}_{12}[6,6,18$, 9, 10].

Superconductivity in $\mathrm{UPd}_{2} \mathrm{Al}_{3}$ was discovered by Geibel et 11] in 1991. The reduction of the Knight shift in NMR[12] and the Pauli limiting of $\mathrm{H}_{c 2}[13]$ indicate spin singlet pairing in this compound. Nodal superconductivity with horizontal nodes has been suggested from the thermal conductivity data 14] and from the c-axis tunneling data of thin film $\mathrm{UPd}_{2} \mathrm{Al}_{3}$ samples [15]. Very recently, McHale et al [16] have proposed $\Delta(\mathbf{k})=\Delta \cos (\chi)\left(\right.$ with $\left.\chi=c k_{z}\right)$ based on a model where the pairing interaction arises from antiparamagnon exchange with $\mathbf{Q}=\left(0,0, \frac{\pi}{c}\right)[17]$. Furthermore, the thermal conductivity data of $\mathrm{UPd}_{2} \mathrm{Al}_{3}$ for a variety of magnetic field orientations have been reported[18]. At first glimpse the experimental data appeared to support the model proposed by McHale et al.

The object of the present paper is to show that an alternative model, i.e. $\Delta(\mathbf{k})=$ $\Delta \cos (2 \chi)$, descibes the thermal conductivity data more consistently. For this purpose we first generalize the universal heat conduction initially proposed in the context of d-wave superconductivity [19, 20] to a variety of nodal superconductors. We limit ourselves to quasi$2 \mathrm{D}$ systems with $\Delta(\mathbf{k})=\Delta f$ and $f=\cos (2 \phi), \sin (2 \phi), \cos \chi, e^{i \phi} \cos \chi, \cos (2 \chi) \sin \chi$, and $e^{i \phi} \sin \chi$. It is found that the in-plane thermal conductivity $\kappa_{x x}$ is independent of $\mathrm{f}$. On the other hand, the out-of-plane thermal conductivity $\kappa_{z z}$ can discriminate different f's. Second, we extend an early study of the angle-dependent thermal conductivity [21] for $\kappa_{y y}$ in a magnetic field rotated in the $\mathrm{z}$-x plane. The comparison of these results with experimental data indicates $\Delta(\mathbf{k})=\Delta \cos (2 \chi)$.

\section{Universal Heat Conduction}

Here we consider the thermal conductivity $\kappa$ in the limit $T \rightarrow 0 K$ in the presence of disorder. It is assumed that the impurities are in the unitary scattering limit [20]. We consider 
the quasi-2D gap functions $\Delta(\mathbf{k})=\Delta f$ with $f=\cos (2 \phi), \sin (2 \phi)$ [d-wave superconductor as in the high- $\mathrm{T}_{c}$ cuprates] $, \cos \chi, e^{i \phi} \cos \chi$ (f-wave superconductor as proposed for $\mathrm{Sr}_{2} \mathrm{RuO}_{4}$ 6] $), \cos (2 \chi), \sin \chi$, and $e^{i \phi} \sin \chi$. Following Ref. 20], the thermal conductivity within the conducting plane is given by

$$
\begin{aligned}
\kappa_{x x} / \kappa_{n}=\kappa_{y y} / \kappa_{n} & =\frac{\Gamma_{0}}{\Delta}\left\langle(1+\cos (2 \phi)) \frac{C_{0}^{2}}{\left(C_{0}^{2}+|f|^{2}\right)^{\frac{3}{2}}}\right\rangle \\
& =\frac{2 \Gamma_{0}}{\pi \Delta \sqrt{1+C_{0}^{2}}} E\left(\frac{1}{\sqrt{1+C_{0}^{2}}}\right)=I_{1}\left(\Gamma / \Gamma_{0}\right)
\end{aligned}
$$

where $\kappa_{n}$ is the thermal conductivity in the normal state when $\Gamma=\Gamma_{0}$, and $\Gamma$ is the quasiparticle scattering rate in the normal state. Here $\langle\ldots$.$\rangle denotes the average over \phi$ and $\chi$, and Eq.(1) tells us that the planar thermal conductivity is independent of the gap functions given above. Also $\Gamma_{0}=\frac{\pi}{2 \gamma} T_{c}=0.866 T_{c}$ and $T_{c}$ is the superconducting transition temperature of the pure system. However, the quasi-particle scattering rate at $\mathrm{E}=0$ is given by $\Delta C_{0}$, and $C_{0}$ is determined by $[20]$

$$
\frac{C_{0}^{2}}{\sqrt{1+C_{0}^{2}}} K\left(\frac{1}{\sqrt{1+C_{0}^{2}}}\right)=\frac{\pi \Gamma}{2 \Delta}
$$

and $\Delta=\Delta(0, \Gamma)$ has to be determined self-consistently as in [20]. Here $\mathrm{K}(\mathrm{k})$ and $\mathrm{E}(\mathrm{k})$ are the complete elliptic integrals. We show $I_{1}\left(\Gamma / \Gamma_{0}\right)$ in Fig.1. Now let us look at the out-of-plane thermal conductivity $\kappa_{z z}$. This is given by

$$
\begin{aligned}
\kappa_{z z} / \kappa_{n} & =\frac{\Gamma_{0}}{\Delta}\left\langle(1-\cos (2 \chi)) \frac{C_{0}^{2}}{\left(C_{0}^{2}+|f|^{2}\right)^{\frac{3}{2}}}\right\rangle \\
& =I_{1}\left(\Gamma / \Gamma_{0}\right)
\end{aligned}
$$

for $f=\cos (2 \phi), \sin (2 \phi)$ and $\cos (2 \chi)$, but

$$
\begin{aligned}
\frac{\kappa_{z z}}{\kappa_{n}} & =\frac{4 \Gamma_{0}}{\pi \Delta \sqrt{1+C_{0}^{2}}}\left(E\left(\frac{1}{\sqrt{1+C_{0}^{2}}}\right)-C_{0}^{2}\left(K\left(\frac{1}{\sqrt{1+C_{0}^{2}}}\right)-E\left(\frac{1}{\sqrt{1+C_{0}^{2}}}\right)\right)\right) \\
& =I_{2}\left(\frac{\Gamma}{\Gamma_{0}}\right)
\end{aligned}
$$

for $f=\cos \chi, e^{ \pm i \phi} \cos \chi$, and

$$
\kappa_{z z} / \kappa_{n}=\frac{2 \Gamma_{0} \Gamma}{\Delta}\left(1-\frac{E\left(\frac{1}{\sqrt{1+C_{0}^{2}}}\right)}{K\left(\frac{1}{\sqrt{1+C_{0}^{2}}}\right)}\right) \equiv I_{3}\left(\frac{\Gamma}{\Gamma_{0}}\right)
$$

for $f=\sin \chi, e^{i \phi} \sin \chi$. These functions are shown in Fig. 1 . 


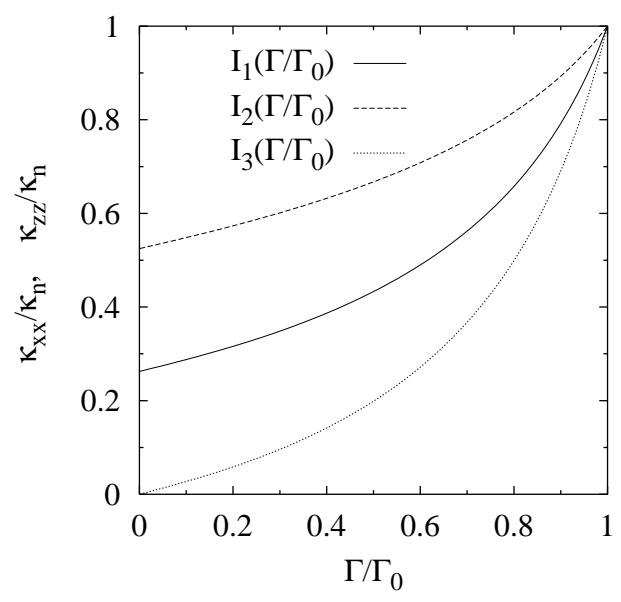

FIG. 1: The functions $I_{1}, I_{2}$ and $I_{3}$.

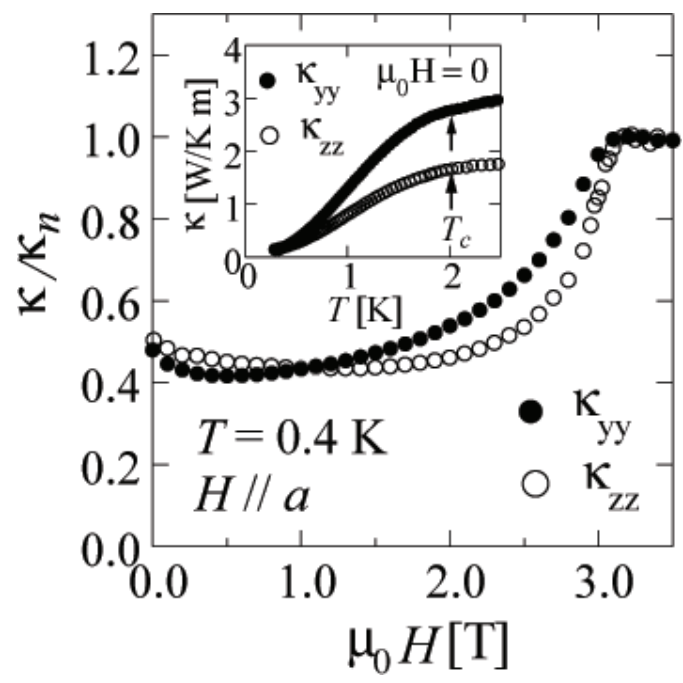

FIG. $2: \kappa_{y y}(H)$ and $\kappa_{z z}(H)$ for $\mathrm{UPd}_{2} \mathrm{Al}_{3}$

In Fig. 2 we show $\kappa_{y y}(H)$ and $\kappa_{z z}$ for $\mathbf{H} \| \hat{z}$ taken for $\mathrm{UPd}_{2} \mathrm{Al}_{3}$ [22]. In particular $\left(\kappa_{00}\right)_{y y}=\left(\kappa_{00}\right)_{z z}$ indicates $\Delta(\mathbf{k}) \sim \cos (2 \chi)$. Of course the effect of the magnetic field is not equivalent to the effect of impurities. But this comparison points to $\Delta(\mathbf{k}) \sim \cos (2 \chi)$ for $\mathrm{UPd}_{2} \mathrm{Al}_{3}$. We note also that for $f=\sin \chi$ and $e^{i \phi} \sin \chi$, there will be no universal heat 
conduction in $\kappa_{z z}$.

\section{Angle-dependent magnetothermal conductivity}

First let us recapture the quasiparticle density of states in the vortex state of nodal superconductors. 44] For simplicity we consider f's with horizontal nodes: $f=$ $e^{i \phi} \cos \chi, \cos \chi, \cos 2 \chi, \sin \chi$ and $e^{i \phi} \sin \chi[21]$. Then the first two f's have nodes at $\chi_{0}= \pm \frac{\pi}{2}$, whereas $f=\cos 2 \chi$ at $\chi_{0}= \pm \frac{\pi}{4}$ and $f=\sin \chi$ and $e^{i \phi} \sin \chi$ at $\chi_{0}=0$.

In an arbitrary field orientation we obtain the quasiparticle density of states

$$
\mathcal{G}(\mathbf{H}) \equiv \frac{N(0, \mathbf{H})}{N_{0}}=\frac{2}{\pi^{2}} \frac{v_{a} \sqrt{e H}}{\Delta} I_{1}(\theta)
$$

for the superclean limit and

$$
\mathcal{G}(\mathbf{H}) \simeq\left(\frac{2 \Gamma}{\pi \Delta}\right)^{1 / 2}\left[\log \left(4 \sqrt{\frac{2 \Delta}{\pi \Gamma}}\right)\right]^{1 / 2}\left(1+\frac{v_{a}^{2} e H}{8 \pi^{2} \Gamma \Delta} \log \left(\frac{\Delta}{v_{a} \sqrt{e H}}\right) I_{2}(\theta)\right)
$$

for the clean limit, where

$$
\begin{aligned}
I_{1}(\theta) & =\left(\cos ^{2} \theta+\alpha \sin ^{2} \theta\right)^{1 / 4} \frac{1}{\pi} \int_{0}^{\pi} d \phi\left(\cos ^{2} \theta+\sin ^{2} \theta\left(\sin ^{2} \phi+\alpha \sin ^{2} \chi_{0}\right)+\sqrt{\alpha} \sin \left(\chi_{0}\right) \cos \phi \sin (2 \theta)\right)^{1 / 2} \\
& \simeq\left(\cos ^{2} \theta+\alpha \sin ^{2} \theta\right)^{1 / 4}\left(1+\sin ^{2} \theta\left(-\frac{1}{2}+\alpha \sin ^{2} \chi_{0}\right)\right)^{1 / 2}\left(1-\frac{1}{64} \frac{\sin ^{2} \theta\left(\sin ^{2} \theta+16 \alpha \sin ^{2} \chi_{0} \cos ^{2} \theta\right)}{\left(1+\sin ^{2} \theta\left(-\frac{1}{2}+\alpha \sin ^{2} \chi_{0}\right)\right)^{2}}\right)
\end{aligned}
$$

and

$$
I_{2}(\theta)=\left(\cos ^{2} \theta+\alpha \sin ^{2} \theta\right)^{1 / 2}\left(1+\sin ^{2} \theta\left(-\frac{1}{2}+\alpha \sin ^{2} \chi_{0}\right)\right) .
$$

Here $\alpha=\left(v_{c} / v_{a}\right)^{2}$ and $\theta$ is the angle $\mathbf{H}$ makes from the $\mathrm{z}$-axis. Then the specific heat, the spin susceptibility and the planar superfluid density in the vortex state in the limit $T \rightarrow 0 K$ are given by [23]

$$
\begin{aligned}
C_{s} / \gamma_{N} T & =\mathcal{G}(\mathbf{H}), \frac{\chi_{S}}{\chi_{N}}=\mathcal{G}(\mathbf{H}), \\
\frac{\rho_{S \|}(\mathbf{H})}{\rho_{S \|}(0)} & =1-\mathcal{G}(\mathbf{H})
\end{aligned}
$$

Similarly the thermal conductivity $\kappa_{y y}$ when the magnetic field is rotated in the $\mathrm{z}$-x plane is given by

$$
\frac{\kappa_{y y}}{\kappa_{n}}=\frac{2}{\pi^{3}} \frac{v_{a}^{2} e H}{\Delta^{2}} F_{1}(\theta)
$$

in the superclean limit and

$$
\frac{\kappa_{y y}}{\kappa_{00}}=1+\frac{v_{a}^{2}(e H)}{6 \pi^{2} \Gamma \Delta} F_{2}(\theta) \log \left(2 \sqrt{\frac{2 \Delta}{\pi \Gamma}}\right) \log \left(\frac{2 \Delta}{v_{a} \sqrt{e H}}\right)
$$


in the clean limit where

$$
\begin{aligned}
& F_{1}(\theta)=\sqrt{\cos ^{2} \theta+\alpha \sin ^{2} \theta}\left(1+\sin ^{2} \theta\left(-\frac{3}{8}+\alpha \sin ^{2} \chi_{0}\right)\right) \\
& F_{2}(\theta)=\sqrt{\cos ^{2} \theta+\alpha \sin ^{2} \theta}\left(1+\sin ^{2} \theta\left(-\frac{1}{4}+\alpha \sin ^{2} \chi_{0}\right)\right)
\end{aligned}
$$

We show in Fig. $3 F_{1}(\theta)$ and $F_{2}(\theta)$ for $\alpha=0.69$ (the value appropriate for $\mathrm{UPd}_{2} \mathrm{Al}_{3}$ ) and
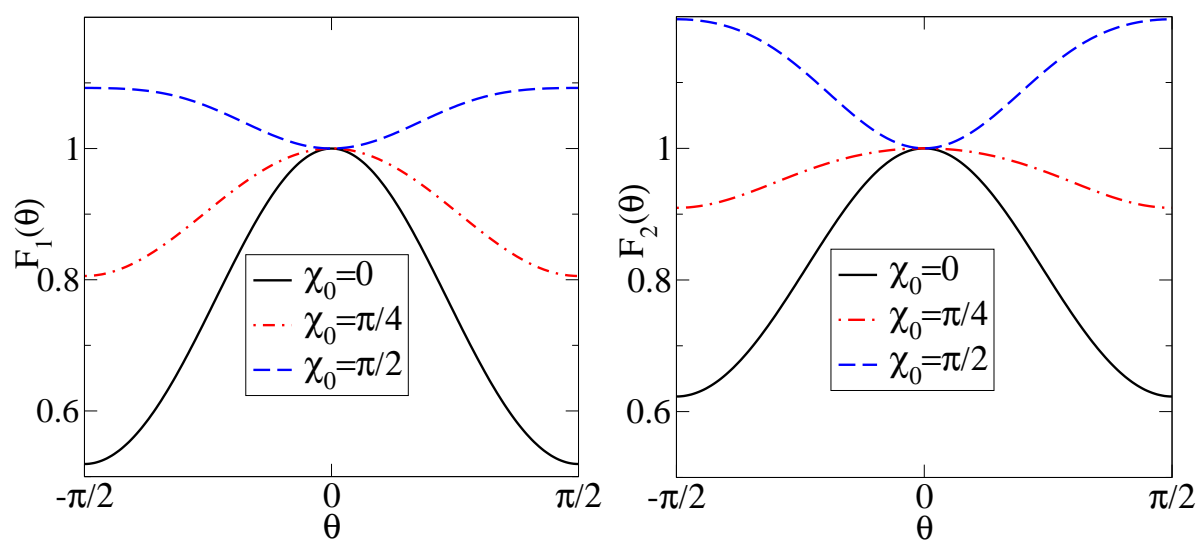

FIG. 3: The angular functions $\mathrm{F}_{1}(\theta)$ (left) and $\mathrm{F}_{2}(\theta)$

$\chi_{0}=0, \frac{\pi}{4}$, and $\frac{\pi}{2}$, which is compared with the experimental data [18] taken at $T=0.4 K$ shown in Fig. 4. Except for the data taken for $H=2.5 T$, the data for $H=0.5 T, 1 T$ and $2 T$ are consistent with $\chi_{0}=\frac{\pi}{4}$, indicating again $f=\cos 2 \chi$. We note also the sign of the twofold term in $\kappa_{y y}$ at $T=0.4 K$ changes sign at $H=0.36 T$. This is consistent with the fact that for $T<v \sqrt{e H}$ the nodal excitations are mostly due to the Doppler shift while for $T>v \sqrt{e H}$ the thermal excitations dominate[24].

\section{Concluding Remarks}

We have analyzed recent thermal conductivity data [18] of $\mathrm{UPd}_{2} \mathrm{Al}_{3}$ from 2 perspectives: universal heat conduction and the angle-dependence. The present study indicates $\Delta(\mathbf{k})=$ $\Delta \cos (2 \chi)$. This is different from the conclusion reached in Ref.[18]. Also we have extended the universal heat conduction for a class of superconducting order parameters $\Delta(\mathbf{k})$, which will be useful for identifying the gap symmetry of new superconductors such as $\mathrm{URu}_{2} \mathrm{Si}_{2}$ and $\mathrm{UNi}_{2} \mathrm{Al}_{3}$.

Furthermore, we have worked out the expressions for $\kappa_{y y}$ when the magnetic field is rotated within the $\mathrm{z}-\mathrm{x}$ plane. The angle dependence of $\kappa_{y y}$ is extremely useful to locate the nodal lines when all nodal lines are horizontal. Perhaps $\kappa_{y y}$ in $\mathrm{Sr}_{2} \mathrm{RuO}_{4}$ will help to identify the precise position of the horizontal nodal lines in $\Delta(\mathbf{k})$, if a further study of 


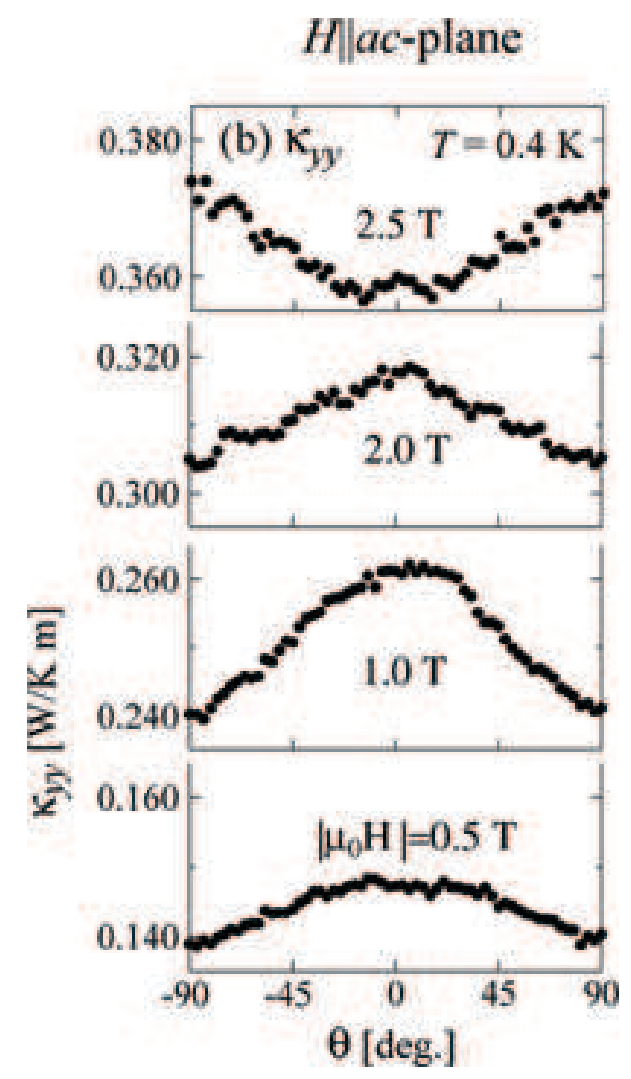

FIG. 4: Angular-dependent magnetothermal conductivity $\kappa_{y y}$ of $\mathrm{UPd}_{2} \mathrm{Al}_{3}$

nodal lines is necessary. Also after $\mathrm{UPt}_{3}$ and $\mathrm{UPd}_{2} \mathrm{Al}_{3}$ we expect many of the U-compound superconducting energy gaps have horizontal lines.

\section{Acknowledgments}

We thank Balazs Dora, Hae-Young Kee, Peter Thalmeier, and Attila Virosztek for helpful collaborations on related subjects.

[1] F. Steglich, J. Aarts, C.D. Bredl, W. Lieke, D. Meschede, W. Franz and H. Schäfer, Phys. Rev. Lett. 43, 1892 (1979).

[2] M. Sigrist and K. Ueda, Rev. Mod. Phys. 63, 239 (1991).

[3] G.E. Volovik, JETP Lett. 58, 469 (1993).

[4] H. Won and K. Maki, cond-mat/0004105.

[5] T. Dahm, H. Won and K. Maki, cond-mat/0006301

[6] K. Izawa et al, Phys. Rev. Lett. 86, 2653 (2001). 
[7] K. Izawa et al, Phys. Rev. Lett. 87, 57002 (2001).

[8] K. Izawa et al, Phys. Rev. Lett. 88, 27002 (2002).

[9] K. Izawa et al, Phys. Rev. Lett. 89, 137006 (2002).

[10] K. Izawa et al, Phys. Rev. Lett. 90, 11701 (2003).

[11] C. Geibel et al, Z. Phys. B 84, 1 (1991).

[12] H. Tou et al, J. Phys. Soc. Jpn. 64,725 (1995).

[13] J. Hessert et al, Physica B 230-232, 373 (1997).

[14] May Chiao, B. Lussier, E. Elleman and L. Taillefer, Physica B 230, 370 (1997).

[15] M. Jourdan, M. Huth and H. Adrian, Nature 398, 47 (1999).

[16] P. MacHale, P. Thalmeier and P. Fulde, cond-mat/0401520.

[17] N. Bernhoeft, Eur. Phys. J. B, 685 (2000).

[18] T. Watanabe et al, cond-mat/0405211.

[19] P. A. Lee, Phys. Rev. Lett. 71, 1887 (1993).

[20] Y. Sun and K. Maki, Europhys. Lett. 32, 335 (1995).

[21] P. Thalmeier and K. Maki, Europhys. Lett. 58, 119 (2002).

[22] T. Watanabe et al (unpublished).

[23] H. Won and K. Maki, Europhys. Lett. 56, 729 (2001).

[24] H. Won and K. Maki, Current Appl. Phys. 1, 291 (2001); also in "Vortices in Unconventional Superconductors and Superfluids", edited by G. E. Volovik, N. Schopohl and R. P. Huebener (Springer, Berlin 2002). 